\title{
Auditory Decision Aiding in Supervisory Control of Multiple Unmanned Aerial Vehicles
}

\author{
Birsen Donmez, M. L. Cummings, Hudson D. Graham \\ Massachusetts Institute of Technology, Cambridge, MA, USA
}

Running title: Auditory aids in supervisory control

Corresponding author

Birsen Donmez

77 Massachusetts Ave, Building 33-407

Cambridge, MA 02139

Telephone: (617)258-5046

Fax: (617)253-4196

bdonmez@mit.edu 


\begin{abstract}
Objectives: This paper investigates the effectiveness of sonification, continuous auditory alert mapped to the state of a monitored task, in supporting unmanned aerial vehicle (UAV) supervisory control. Background: UAV supervisory control requires monitoring each UAV across multiple tasks (e.g., course maintenance) via a predominantly visual display, which currently is supported with discrete auditory alerts. Sonification has been shown to enhance monitoring performance in domains such as anesthesiology by allowing an operator to immediately determine an entity's (e.g., patient) current and projected states, and is a promising alternative to discrete alerts in UAV control. However, minimal research compares sonification to discrete alerts, and no research assesses the effectiveness of sonification for monitoring multiple entities (e.g., multiple UAVs). Method: An experiment was conducted with 39 military personnel, using a simulated setup. Participants controlled single and multiple UAVs, and received sonifications or discrete alerts based on UAV course deviations and late target arrivals. Results: Regardless of the number of UAVs supervised, the course deviation sonification resulted in $1.9 \mathrm{~s}$ faster reactions to course deviations, a $19 \%$ enhancement from discrete alerts. However, course deviation sonification interfered with the effectiveness of discrete late arrival alerts in general, and with operator response to late arrivals when supervising multiple vehicles. Conclusions: Sonifications can outperform discrete alerts when designed to aid operators to predict future states of monitored tasks. However, sonifications may mask other auditory alerts, and interfere with other monitoring tasks that require divided attention. Applications: This research has implications for the design of supervisory control displays.
\end{abstract}

Keywords: Continuous auditory alerts, sonifications, unmanned vehicles, discrete auditory alerts 


\section{INTRODUCTION}

The use of Unmanned Aerial Vehicles (UAVs) is growing in the military, federal, and civil domains. Worldwide, in the next eight years UAVs will be a 15.7 billion dollar industry, where in the United States alone the plan is to acquire 9,420 mini UAVs, 775 tactical UAVs, 570 minimum altitude, long endurance UAVs and 60 Global Hawks (Tsach et al., 2007). UAVs are not just in demand on the battlefield; other government departments seeking the use of UAVs include the Department of Homeland Security and the US Coast Guard for law enforcement and border patrol (DOD, 2007). UAVs are also sought after in support of humanitarian relief efforts, such as with flood regions in the US. In Missouri in May 2007, Air Force Predator UAVs were on standby to offer assistance in flood recovery efforts (Arana-Barradas, 2007).

From January to October 2007, the number of hours for mission sorties doubled for UAVs in the Air Force, creating a staffing crisis. A technological solution proposed by many Department of Defense senior leaders and researchers to alleviate this crisis is to move from a team of operators controlling one UAV to having a single operator controlling multiple UAVs (Barbato, Feitshans, Williams, \& Hughes, 2003; Culbertson, 2006; Tsach et al., 2007). To achieve this force multiplication, vehicles will have to be more autonomous, placing humans more in a supervisory role. The role of the human will switch from attending to low-level tasks like manually flying the aircraft to higher-level tasks such as monitoring and generating contingency courses of action. Thus, research is needed to understand how best to aid and integrate the human operator into a system of semi-autonomous UAVs, supporting such supervisory control tasks. UAV displays are predominantly visual displays and are typically mounted in mobile trailers, truck beds, or backpacks (McCauley \& Matsangas, 2004). Due to 
potential information overload and visual saturation of UAV operators, offloading some perceptual processing to other senses can be beneficial.

When there is an overload of information, utilizing multiple sensory channels can effectively increase the amount of information processed (Wickens, Lee, Liu, \& Becker, 2004). Moreover, dual-coding of information can speed processing (Miller, 1991). In a meta-analysis of 43 previous studies, Burke et al. (2006) concluded that dual coding information on the visual and auditory, or visual and tactile modalities enhances performance, while potentially decreasing workload. Currently, discrete auditory alerts are used in UAV operations as a supplement to visual information, and research shows that providing audio alerts to UAV operators enhances their performance in monitoring automated tasks, as well as their overall mission performance (Dixon, Wickens, \& Chang, 2005). A notable benefit for auditory presentation is its effectiveness at representing simple events occurring in time and requiring immediate action (Deatherage, 1972; Sorkin, 1987). Because of its omnipresence and the ability to help operators stay in key control loops via preattentive information processing, audio is usually the preferred sense for presenting a warning (Simpson \& Williams, 1980; Sorkin, 1987; Woods, 1995). However, discrete alerts are also prone to masking, and may be missed by the operator. Moreover, given their short duration, discrete alerts cannot relay detailed information on the monitored task's state.

One alternative is to continuously present auditory information in order to potentially leverage preattentive processing of task relevant information, so that the operator can quickly determine the task's current state, as well as projected states. Sonifications are continuous auditory alerts mapped to the state of the monitored task (Kramer, 1994). A pulse oximeter is an example for a sonification, which indicates the oxygen saturation in arterial blood and the heart 
rate through the pitch and the repetition of a continuous series of beeps, respectively. Watson and Sanderson (2004) suggest that, in comparison to discrete alerts, sonifications can better provide background information about changed states without a major disruption of attentional focus. Research has shown sonifications to be beneficial in medical and assistive technologies, scientific visualizations, engineering analysis, emergency alerts, and aircraft cockpits (Barrass \& Kramer, 1999).

In anesthesiology, combined visual and sonification displays have been shown to outperform each alone. For example, Loeb and Fitch (2002) found that a combined visual and sonification display resulted in quicker reaction times than each alone for detecting abnormalities in heart and respiratory rates. In a similar study, Watson and Sanderson (2004) asked participants to divide their attention between monitoring for an abnormal respiratory event and performing an arithmetic true-false task. Regardless of the display modality, the performance was the same for monitoring, but that for the time-shared task, participants performed best with the combined display. While these studies showed benefits to combined visual and sonification displays, it is not clear how sonifications compare with discrete audio alerts. Only one study we know of compared sonifications to discrete audio (Pacey \& MacGregor, 2001). Participants were provided with sonifications for monitoring file downloads while typing poems. The sonification results were mixed, suggesting an enhancement in the monitored task but also a larger interference with the time-shared task. It is also unclear if sonifications would be effective for monitoring multiple entities (e.g., multiple patients or UAVs) since there is no existing research investigating this issue.

The purpose of the current experiment was to identify if sonifications better aid human supervision of UAV operation when compared to discrete audio alerts, and if the number of 
UAVs under supervision, that is, the number of entities monitored, influences the effectiveness of these auditory alerts. Another objective was to assess if sonifications interfered with the effectiveness of discrete alerts provided for other time-shared monitoring tasks per each UAV, which included course conformance and on-time target arrival.

\section{METHOD}

In this experiment, operators supervised either single or multiple UAVs using a simulated setup. The overall goal was to engage as many pre-planned targets as possible, while making sure that the UAVs arrived back at the base safely. Operator tasking included arming and firing on targets with various priorities, while monitoring each UAV's flight of path, event timeline, and radio traffic. The UAVs also occasionally required navigation re-planning due to external influences.

\section{Participants}

Thirty nine US military personnel ( 6 females, 33 males) between the ages of 20-41 (mean=25.0, $\mathrm{SD}=5.6$ ) completed the study. Overall, participants had a combined experience of 223 years of active duty military service (mean=5.7, $\mathrm{SD}=4.8$ ). There were three Navy midshipmen (college education required), one Air Force staff sergeant (high school degree required), and 35 officers from the Army/Navy/Air Force (college education required at the least). Participants had familiarity with UAVs but none were UAV operators. There were a total of three pilots, and most of the 28 junior officers tested will be future military pilots, from which the military selects its UAV operators. Participants were screened for hearing impairments and colorblindness, and were compensated $\$ 10 /$ hour. 


\section{Apparatus}

The experiment was carried out in the Multiple Autonomous Unmanned Vehicle Experimental (MAUVE) test bed, which is a two screen interface that allows the simulation of any number of unmanned vehicles conducting strike operations in hostile environments (Figure 1).

$<<$ Insert Figure 1 >>

The test bed provides a map for geo-spatial tracking of UAVs on predetermined flight paths, along with an interaction control panel for UAV control inputs (Figure 1a). Control inputs include arming and firing a UAV to destroy targets, directing specific path changes by either skipping a target in order to get a UAV on schedule or resetting a UAV's onboard navigation if the UAV drifts off course, and acknowledging radio calls. A timeline and a scheduling decision support visualization (DSV) tool for each UAV, along with a message and a health and status monitoring box are also provided (Figure 1b). A timeline display presents a schedule for each UAV, including target arrivals and phase of flight. The DSV aids the operator in responding to late target arrivals (Brzezinski, Seybold, \& Cummings, 2007). The chat window displays text messages from other simulated operators that appear in response to scenario events or actions taken by operators (e.g., a message indicating that a delay requested in response to a late target arrival is accepted).

MAUVE was run on a multi-modal workstation (Figure 2), a four screen computer display (Osga et al., 2002). The three screens across the top are 21 inches (1280x1024), and the bottom screen is 15 inches $(1024 \times 768)$. Throughout the experiment, the left screen showed the mission objectives and priorities, and the bottom displayed the color-coding for UAV flight 
phases (e.g., blue for loitering). The center screen contained the MAUVE map, and the right screen included the MAUVE timeline.

$<<$ Insert Figure 2>>

A Sensimetrics HDiSP headset (Figure 2) was used to present the auditory alerts and the auditory secondary task used in the experiment (Graham \& Cummings, 2007). This headset provides ambient noise attenuation ranging from 35 to $40 \mathrm{db}$ for the spectrum between $500 \mathrm{~Hz}$ and $8 \mathrm{kHz}$.

\section{Experimental Tasks}

As previously stated, the mission objective was to ensure that the UAVs returned to base safely by a specified time, and that correct targets were engaged during specified windows of opportunity, i.e., the projected time available to engage a target. Two types of events occurred that complicated this mission objective, course deviation and late target arrivals. As in real operations, unexpected head or crosswinds could cause UAVs to slow their speed or drift off course, resulting in course deviations and possible late arrivals to targets. Therefore, participants were instructed to monitor for and respond to these events. In each test scenario, participants were presented with a total of four course deviations and four late target arrivals. Anytime the UAV completely departed from its course line, that is, the UAV icon was no longer touching the line, it was considered to have deviated (Figure 3a). The correct intervention in this case was to reset the onboard navigation in the UAV. The visual representation for a late arrival is presented in Figure 3b. The objects on this timeline move left as the UAV travels further on its course. A black rectangle on the timeline projects arrival to the target, which should either coincide with the predetermined window of opportunity or before, if loitering. This rectangle changes from black to red when the UAV is late (i.e., black rectangle moves past the window of opportunity). 
If the UAV is late to a target, then the window of opportunity starts to shrink. If the window of opportunity disappears, then it is too late to engage the target and the target is missed.

$$
<<\text { Insert Figure 3>> }
$$

In order to address the late arrivals, participants were instructed to skip low priority targets. For medium priority targets, operators were given the option to use the DSV. For high priority targets, participants were required to employ DSV before requesting a delay from a simulated higher authority or skipping the target. In Figure 3c, the DSV illustrates that the UAV is late to a high priority target, and if a delay is granted, the UAV would be late to another high priority target. The probability of the granting of a delay request is also presented. If the delay was granted, the UAV was no longer late to that target.

As a secondary workload task, participants were instructed to monitor a recording of continual air traffic radio chatter for the word "Push", which occurred 62 times in a 30 minute session, with an average of $27 \mathrm{~s}$ between occurrences. To acknowledge the radio call, participants clicked the "Acknowledge Push" button (Figure 1a).

\section{Experimental Design and Independent Variables}

The experiment was a $2 \times 2 \times 2$ mixed factorial design with two between subject conditions: course deviation alert type (discrete, sonification), and late arrival alert type (discrete, sonification). The number of UAVs under control (single, multi) was a within-subject condition presented in a counterbalanced fashion. The subjects were nested under the interaction of course deviation alert type and late arrival alert type (Table 1) and were randomly assigned. The alert types were crossed in order to assess how operators respond to one alert in the presence of another, in particular to determine if discrete alerts are masked by sonifications. A no-alert 
baseline was not included since dual coding is the standard of care in UAV supervisory control, where visual information is supplemented with discrete audio alerts.

\section{$<<$ Insert Table 1 >>}

Number of UAVs. The multi UAV condition consisted of four UAVs. For the single and multi UAV conditions, participants were presented with the same number and types of events at the same time. There were eight total events, which were either all performed by the single UAV, or in the case of the multi UAV condition, were spread across the UAVs. The intent was to control for the task load as much as possible, in order to truly assess the effects of sonifications for monitoring multiple entities compared to monitoring a single entity.

Alert types. The discrete alerts used in the experiment (for both course deviations and late arrivals) were chosen to closely reflect those used in actual UAV ground control stations (GCS). In particular, the Army's Shadow GCS was used as the model. The sound intensity levels for both the discrete alerts and the sonifications were set in pilot testing to ensure that the different levels were audible and distinguishable from other levels and alerts. A total of twelve participants with no hearing impairments were tested in the pilot study.

Course deviation alert type. The discrete alert consisted of a beep at $1000 \mathrm{~Hz}$, which lasted $\sim 8 \mathrm{~ms}$, and played once when a UAV drifted off-course, as depicted in Figure 3a. The sonification represented both the existence and severity of the UAV course deviations. It was designed to create a semantic relationship between the audio and the course deviation event, which in real operations would be caused by unexpected crosswinds. It was a sound that increased in intensity and oscillated faster as the UAV deviated further off course. It was similar to how wind sounds while driving in a convertible car. Because the sonification was continuous, the sonification changed as the UAV drifted off course even when it was not considered to have 
completely departed. The alert was generated by applying comb filters to a mix of pink noise and the ambient signal. The mix ranged from 0.2 pink noise for low deviation to 0.9 pink noise for high deviation. The comb filters had a base delay of $0.2 \mathrm{~ms}$, with a $50 \%$ mix of the base signal and a feed forward delay. The delay values were then oscillated to create a periodic signal. The alert played continually to provide an auditory image of UAV path position. As a UAV drifted further off course, the frequency of oscillation of the comb filter delay decreased from $17 \mathrm{~Hz}$ to 4.5 Hz, and the depth of oscillation increased from $0.2 \mathrm{~ms}$ to $0.7 \mathrm{~ms}$. When the operator reset the onboard navigation, the UAV immediately returned to its course with the sonification matched to indicate this. In the multi UAV condition, the audio alert played for whichever UAV was furthest off course.

Late arrival alert type. The discrete alert consisted of a beep at $415 \mathrm{~Hz}$, which lasted $\sim 18$ ms, and played once when a UAV was projected to be late to a target. In contrast to the course deviation event, the late arrival event was discrete in nature (i.e., the UAV was either late to a target or not), thus, a tiered sonification was used for late arrivals. The degree of lateness was not conveyed in the sonification since this does not match operators' expectations, who are generally concerned with ensuring UAVs get to targets at predetermined times, not as close to a time as possible.

The sonification consisted of discrete harmonic signals that continuously played to indicate a projected late-arrival at a target until the operator took corrective action, or until the UAV continued past the target when the operator failed to take action. It sounded like continuously playing a chord on an organ and changing to a higher pitch note if the UAV was predicted to be late to a target. Three different pitches conveyed different urgency levels for late arrivals to low, medium, and high priority targets. The harmonic signals were composed of five 
Formant filters that were applied to a mix of pink noise and the ambient signal. During the condition of no late-arrivals, a baseline audio was generated with two filters set at $261.6 \mathrm{~Hz}$, two filters set at $329.4 \mathrm{~Hz}$, and one filter set at $392.0 \mathrm{~Hz}$ (a major C triad with extra balance in the root and third). If the UAV was late to a low priority target, a signal was generated with two filters set at $261.6 \mathrm{~Hz}$, two filters set at $311.1 \mathrm{~Hz}$, and one filter set at $392.0 \mathrm{~Hz}$ (a minor C triad with extra balance in the root and third). If the UAV was late to a medium priority target, a signal was generated with three filters set at $293.6 \mathrm{~Hz}$ and two filters set at $415.3 \mathrm{~Hz}$ (a tritone interval with a D root). If the UAV was late to a high priority target, a signal was generated with three filters set at $369.9 \mathrm{~Hz}$ and two filters set at $523.3 \mathrm{~Hz}$ (a tritone interval with an F\# root). As the priority increased, the pink noise mix also increased, from 0.25 for the baseline, to $0.7,0.8$, and 1.0 for the three priority levels.

\section{Procedure}

Each participant experienced a 60-70 minute training session, followed by a 70-80 minute test session, and lastly a 10 minute post-test survey. During training, participants

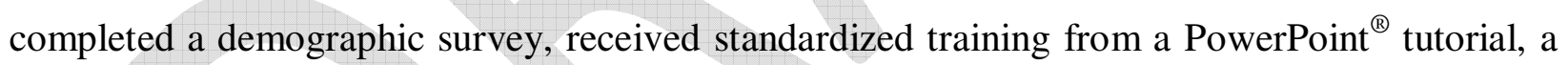
hearing test, audio training for the specific audio test condition where they learned the meaning of different alerts, and a thirty minute practice test session with the multi UAV scenario, including a trial run of the NASA Task Load Index (TLX) (Hart \& Staveland, 1988). Each participant then completed both the single and the multi UAV scenarios, each followed by the administration of NASA TLX.

For the hearing test, participants listened to tones ranging from $40 \mathrm{dBSPL}$ to $16 \mathrm{dBSPL}$, with 5 frequencies $(500 \mathrm{~Hz}, 1,2,4$, and $8 \mathrm{kHz}$ ), and acknowledged how many beeps were heard in each ear and each frequency. The range tested ensured participants could hear to within $3 \mathrm{~dB}$ 
of the noise floor of the headset in normal laboratory conditions. Based on the method of descending limits, the test was designed so that hearing 5 beeps or more at each frequency showed adequate hearing (Snodgrass, 1975; Wundt, 1902).

\section{Dependent Variables}

Primary task performance variables included the number of missed course deviations, reaction time to correct course deviations, number of missed late arrivals, and reaction time to correct projected late arrivals. The number of push call responses was analyzed to assess participants' secondary task workload. Subjective workload was assessed using NASA TLX. Since there was a negligible amount of physical demand in this experiment, participants were told not to rate physical demand, which was not included in the final score.

\section{RESULTS}

The analyses were performed with mixed linear models; with the random subject term nested under the interaction of course deviation alert type and late arrival alert type. An unstructured covariance matrix was assumed in order to model variance heterogeneity, $\alpha=.05$. As for the demographics information, years of active duty military service was not a statistically significant predictor of the dependent variables, and hence was not included in the final models. Level of formal education and gender were not considered as covariates since the former was fairly consistent among participants and the latter was highly unbalanced.

Course deviations. Out of the 312 course deviations presented, nine were missed. Of these nine, six were missed by participants with discrete alerts for course deviations and late arrival sonifications.

Statistical analyses were conducted on the average value of the four course deviation reaction times observed in each scenario. The course deviation alert type had a significant effect 
on reaction times $(F(1,35)=8.47, p=.006)$ (Figure $4 \mathrm{a})$. Sonifications resulted in $1.90 \mathrm{~s}$ faster average course deviation reaction time than discrete alerts $(t(36)=-2.91, p=.006)$. The number of UAVs controlled had only a marginal effect, suggesting a $0.87 \mathrm{~s}$ increase in average reaction times with the multi UAV condition $(F(1,35)=3.22, p=.08)$.

$<<$ Insert Figure 4>>

Late target arrivals. All participants responded to all four late arrivals presented, by either skipping a target or requesting a delay before the window of opportunity disappeared. In order to meet statistical modeling assumptions, a logarithmic transformation (natural logarithm) was performed on the average reaction times (Figure 4b).

Number of UAVs $(F(1,36)=21.35, p=<.0001)$ and its interaction to course deviation alert type $(F(1,36)=4.86, p=.03)$ were significant. When operators were presented with course deviation sonifications, their response to late arrivals degraded significantly as they switched from supervising one UAV to four UAVs $(t(36)=-4.89, p<.0001)$. This effect was not observed for discrete course deviation alerts $(t(36)=-1.69, p=.10)$. Moreover, for single UAV condition, there were no differences between the two course deviation alert types $(t(36)=0.43, p=.67)$, whereas for multi UAV condition, operators who received course deviation sonifications took longer to respond to late arrivals when compared to the operators who received discrete course deviation alerts $(t(35)=3.21, p=.003)$. Therefore, course deviation sonifications interfered with late arrival response in multi UAV supervisory control, but not in single UAV supervisory control.

Further, the interaction of course deviation alert type and late arrival alert type $(F(1,35)=4.27, p=.046)$ was significant for $\log ($ late arrival reaction times). In particular, the combination of course deviation sonifications and discrete late arrival alerts resulted in 
significantly worst response to late arrivals. Therefore, course deviation sonifications interfered with the effectiveness of discrete alerts provided for late arrivals. There were no significant differences for late arrival response among the other three course deviation - late arrival alert combinations.

Workload and post-experiment responses. There were no significant effects for NASA TLX scores, or for the number of push call responses (i.e., secondary task response). Thus, there is no statistical evidence suggesting a perceived workload or spare mental capacity difference between the single and multi UAV scenario, or for the audio alert type. This non-significance can be due to multiple factors, such as the lack of an actual difference between the conditions or the potential insensitivity of the secondary task measure to assess the workload of the primary task.

The majority of the participants (92\% for late arrival and $85 \%$ for course deviation alerts) commented that the audio alerts worked well in conjunction with the visual display to better enhance their awareness. A key concern raised about the discrete audio alerts was that the alerts were hard to distinguish from background noise. This was indicated by $66 \%$ of the participants who were exposed to a discrete alert. A common feeling was that the discrete alert would be more effective if it were longer and more prominent. The sonifications were generally appreciated as situational enhancers and attention managers, but some felt they were potentially fatiguing. Ten percent of the participants exposed to course deviation sonifications suggested that this additional alert be silenced throughout the bulk of the mission, and activated during particular parts of the mission. Interestingly, all of the negative comments regarding course deviation sonifications came from participants who were also exposed to late arrival sonifications. 


\section{DISCUSSION}

An experiment was conducted to evaluate sonifications in a supervisory control setting, specifically monitoring and responding to UAV course deviations and late arrivals to targets. The results showed that regardless of the number of UAVs supervised, sonifications promoted better performance for course deviations, representative of continuous system states, when compared to discrete alerts. With sonifications, participants responded on average $1.9 \mathrm{~s}$ faster to course deviations than participants with the discrete audio alerts; a 19\% decrease in reaction times. In aviation $1.9 \mathrm{~s}$ can be critical, particularly in UAV operations where operators deal with time lags in remote vehicle control. However, there were no observed benefits to sonifications signaling late target arrivals, which also has a temporal continuous component, but is more representative of a discrete state (i.e., late or not late).

Course deviation sonifications were mapped to a UAV's distance from its expected course. This enabled the operators to immediately assess a UAV's current position, but also aided them to project its near future position. In contrast, late arrival sonifications changed in a step-wise fashion, that is, the sonification changed from signaling "not late" to signaling "late to a target" instantaneously, since this is a discrete event. Thus, the main difference between course deviation and late arrival alerts was that the late arrival sonifications did not aid operators in projecting future task states. Arguably, degree of lateness could have been conveyed in the sonification. However, contextually this does not match operators' expectations. As stated in the experimental design section, operators are generally concerned with ensuring UAVs get to targets at predetermined times, not as close to a time as possible. From an information-theory perspective, a tiered sonification conveys less information than an alert representing a larger number of states (in this study, the course deviation sonification represented infinitely many 
states). From the perspective of human perception, the reduction of possible state-changes would affect the operators' noticing a transition from one state to another, and thus may be less apt to pre-attentively capture operators' attention. These provide potential explanations for the lack of observed benefits for late arrival sonifications. Another possible explanation is the specific characteristics of the alerts and sonifications used in this study. Further research is needed to identify the underlying reasons for the lack of benefits observed for tiered-sonifications.

While this study showed sonifications enhanced response to course deviations, the sonifications also appeared to interfere with the effectiveness of discrete late arrival alerts. This suggests a possible masking problem where the sonifications saturated the audio channel to a point that the more traditional beeps for late arrivals were not heard. The discrete alerts used in this study may have been particularly prone to masking since they were based on alerts used in current UAV operations, and were pure tones with no harmonics, pattern, or repetition. One possible way to mitigate such masking effects is to avoid alerts that are pure tones (Patterson, 1990). In the military, UAVs are operated remotely from either local or global control platforms with fluid atmospheres of noise and activity where auditory alerts may be masked, so this is an important possible limitation, particularly for legacy systems.

Course deviation sonifications also appeared to interfere with late arrival responses in multi-UAV supervisory control. When operators were presented with course deviation sonifications, their responses to late arrivals degraded as they switched from supervising one UAV to four UAVs. Given the equal task load design for single and multi UAV conditions, the prominent difference between these conditions was the higher level of divided attention required in the multi UAV case. In the multi UAV condition, the operators not only had to divide their attention between monitoring two tasks (i.e., course deviations and late arrivals), but also had to 
do this across multiple entities (i.e., four UAVs). Therefore, this finding suggests that sonifications may potentially interfere with response to time-shared monitoring tasks when a high level of divided attention is required from the operator.

In their sonification study, Pacey and MacGregor (2001) also observed an enhancement in the monitored task (monitoring a file download) but a degradation in the time-shared task (typing a poem), whereas Watson and Sanderson (2004) observed an enhancement in the timeshared task (arithmetic true-false) with no change in monitoring performance (monitoring a patient's respiration). However, in contrast to our study, the time shared tasks used in these previous studies, typing poems and performing mental arithmetic, did not require monitoring. Moreover, these previous studies did not require the participants to monitor multiple entities, that is, multiple patients and multiple files. Thus, the results obtained are not directly comparable to our findings.

This divided attention problem may be explained in part by alert reference issues that are problematic when supervising multiple similar entities. More specifically, in the multiple UAV case, the auditory alerts, both discrete and sonification-based, did not indicate which UAV they were referring to, so the operators had to visually identify the problem UAV. It is possible that conveying specific UAV information with the audio alert may promote faster response. However, the operators may not be able to distinguish different alerts used for individual UAVs, which would require greater reliance on memory or a natural mapping between different sounds and specific UAVs, which is unlikely. Previous research has shown that a nonspecific general system aural alarm provides performance enhancement over individual alarms, particularly when accompanied by a visual cue (Cummings et al., 2007). Thus, the use of a single aural alert to 
represent a problem among many candidates is justified, however, determining if and how many alerts can be effectively combined in the multi-UAV setting is an area of future research.

Furthermore, some participants in the experiment noted fatigue with long term exposure to sonifications. One system integration solution is to limit exposure and only use sonifications under high workload, or when one of the monitored tasks reaches a cautionary state. Future research should investigate adaptive displays which can add or remove audio aids as required by the operator/system state.

Lastly, while sonification may be a performance enhancer, it is also important to assess the research and development, system deployment, and maintenance costs associated with fielding this new technology. For example, a shortcoming in the use of headphones for the presentation of the sonifications is the isolation of the operator from outside communication (Tannen, 1998). Military operators will often only wear one half of the headset because the headset on both ears isolates them from inter-team interaction. It is important to make sure that any integration of a headset does not interfere with critical work environment constraints, especially those in a team setting.

\section{ACKNOWLEDGMENTS}

This research was sponsored by the US Army through a Small Business Innovation Research led by Charles River Analytics, Inc. Special thanks is extended to Adam Fouse and Dr. Tom von Wiegand for their help in the development of auditory alerts, and to Teresa Pontillo and Brian Malley, undergraduate researchers, who assisted in the data gathering. 


\section{REFERENCES}

Arana-Barradas, L. A. (2007). Predators ready to aid Missouri flood victims. Air Force Link Retrieved September 10, 2008, from http://www.af.mil/news/story.asp?storyID=123053041

Barbato, G., Feitshans, G., Williams, R., \& Hughes, T. (2003). Unmanned combat air vehicle control and displays for suppression of enemy air defenses. Paper presented at the 12th International Symposium on Aviation Psychology, Dayton, $\mathrm{OH}$.

Barrass, S., \& Kramer, G. (1999). Using sonification. Multimedia Systems, 7(1), 23-31.

Brzezinski, A. S., Seybold, A. L., \& Cummings, M. L. (2007). Decision support visualizations for schedule management of multiple unmanned aerial vehicles. In Proceedings of AIAA Infotech Aerospace Conference. Rohnert Park, CA: AIAA.

Burke, J. L., Prewett, M. S., Gray, A. A., Yang, L., Stilson, F. R. B., Coovert, M. D., et al. (2006). Comparing the effects of visual-auditory and visual-tactile feedback on user performance: a meta-analysis. In Proceedings of the 8th International Conference on Multimodal Interfaces (pp. 108-117).

Culbertson, E. (2006). COMUSAFE: Unmanned Aircraft Key to Future Decision Superiority. Retrieved March 18, 2008, from http://www.af.mil/news/story.asp?id=123029520

Cummings, M. L., Kilgore, R. M., Wang, E., Tijerina, K., \& Kochhar, D. S. (2007). Effects of single versus multiple warnings on driver performance. Human Factors, 49(6), 10971106. 
Deatherage, B. H. (1972). Auditory and other sensory forms of information processing. In H. P. VanCott \& R. G. Kinkade (Eds.), Human Engineering Guide to Equipment Design. Washington, D. C.: American Institutes for Research.

Dixon, S., Wickens, C. D., \& Chang, D. (2005). Mission control of multiple unmanned aerial vehicles: a workload analysis. Human Factors, 47, 479-487.

DOD. (2007). Unmanned systems roadmap (No. 2007-2032): Office of the Secretary of Defense, Washington, D.C.

Graham, H. D., \& Cummings, M. L. (2007). Assessing the impact of auditory peripheral displays for UAV operators (No. HAL2007-09): Humans and Automation Laboratory, MIT.

Hart, S. G., \& Staveland, L. E. (1988). Development of NASA-TLX (Task Load Index): results of empirical and theoretical research. In P. Hancock \& N. Meshkati (Eds.), Human Mental Workload (pp. 139-183). Amsterdam, The Netherlands: North Holland B. V.

Kramer, G. (1994). Some organizing principles for representing data with sound. In G. Kramer (Ed.), Auditory Display: Sonification, Audification and Auditory Interfaces. SFI Studies in the Sciences of Complexity, Proceedings Volume XVIII (pp. 185-222). Reading, MA: Addison Wesley.

Loeb, R. G., \& Fitch, W. T. (2002). A laboratory evaluation of an auditory display designed to enhance intraoperative monitoring. Anesthesia and Analgesia, 94, 362-368.

McCauley, M. E., \& Matsangas, P. (2004). Human Systems Integration and Automation Issues in Small Unmanned Aerial Vehicles (No. NPS-OR-04-008). Monterey, CA: Naval Postgraduate School. 
Miller, J. (1991). Channel interaction and the redundant-targets effect in bimodal divided attention. Journal of Experimental Psychology: Human Perception and Performance, 17, 160-169.

Osga, G., Van Orden, K., Campbell, N., Kellmeyer, D., \& Lulue, D. (2002). Design and evaluation of warfighter task support methods in a multi-modal watchstation (No. 1874): SPAWAR, San Diego.

Pacey, M., \& MacGregor, C. (2001). Auditory cues for monitoring a background process: a comparative evaluation. In Proceedings of the Human Computer Interaction: INTERACT Conference. Tokyo, Japan.

Patterson, R. D. (1990). Auditory warning sounds in the work environment. Philosophical Transactions of the Royal Society of London Series, Series B, Biological Sciences, $327(1241), 485-492$.

Simpson, C., \& Williams, D. H. (1980). Response time effects of alerting tone and semantic context for synthesized voice cockpit warnings. Human Factors, 22, 319-330.

Snodgrass, J. G. (1975). Psychophysics. In B. Scharf (Ed.), Experimental Sensory Psychology. Glenview, IL: Scott Foresman \& Co.

Sorkin, R. D. (1987). Design of auditory and tactile displays. In G. Salvendy (Ed.), Handbook of Human Factors (pp. 549-576). New York: Wiley.

Tannen, R. S. (1998). Breaking the sound barrier: designing auditory displays for global usability. Paper presented at the 4th Conference on Human Factors \& the Web, Basking Ridge, New Jersey. 
Tsach, S., Peled, A., Penn, D., Keshales, B., \& Guedj, R. (2007). Development trends for next generation UAV systems. Paper presented at the AIAA 2007 Conference and Exhibit, Rohnert Park, California.

Watson, M., \& Sanderson, P. (2004). Sonification supports eyes-free respiratory monitoring and task time-sharing. Human Factors, 46(3), 497-517.

Wickens, C. D., Lee, J. D., Liu, Y., \& Becker, S. G. (2004). An Introduction to Human Factors Engineering (2nd ed.). Upper Saddle River, New Jersey: Pearson Education, Inc.

Woods, D. D. (1995). The alarm problem and direct attention in dynamic fault management. Ergonomics, 38, 2371-2393.

Wundt, W. M. (1902). Principles of Physiological Psychology. London: Swan Sonnenschein \& Co. Lim.

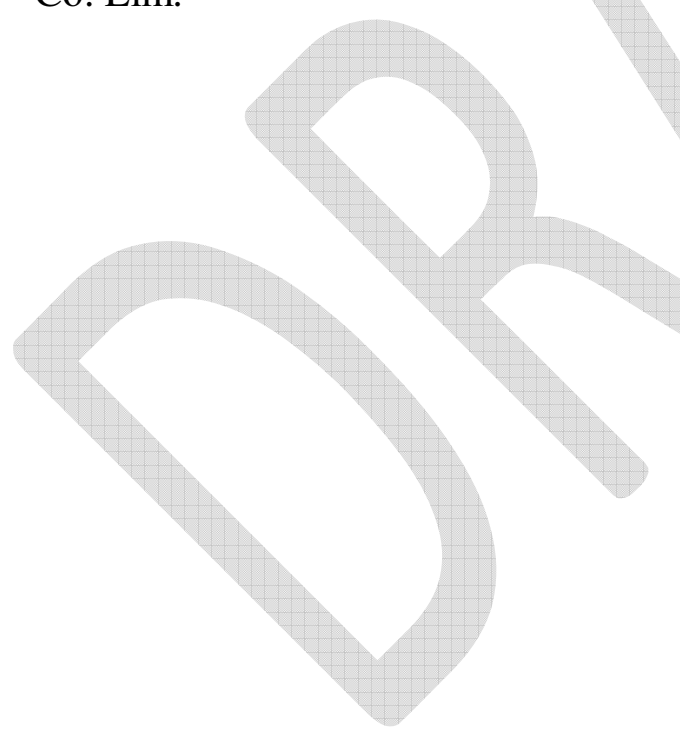




\section{Table 1. Experimental design}

\begin{tabular}{llll} 
& & \multicolumn{2}{c}{ Number of UAVs } \\
\cline { 3 - 4 } Late arrival alert type & Course deviation alert type & Single & Multiple (four) \\
\hline \multirow{2}{*}{ Discrete } & Discrete & $\mathrm{n}=9$ (group A) & $\mathrm{n}=9$ (group A) \\
& Sonification & $\mathrm{n}=10$ (group B) & $\mathrm{n}=10$ (group B) \\
\hline \multirow{2}{*}{ Sonification } & Discrete & $\mathrm{n}=10$ (group C) & $\mathrm{n}=10$ (group C) \\
& Sonification & $\mathrm{n}=10$ (group D) & $\mathrm{n}=10$ (group D) \\
\hline
\end{tabular}




\section{LIST OF FIGURES}

Figure 1. Multiple Autonomous Unmanned Vehicle Experimental (MAUVE) test bed (a) map display (b) timeline display

Figure 2. The multi-modal workstation

Figure 3. (a) A course deviation event (b) a late arrival event (c) decision support visualization for late arrivals

Figure 4. Box plots for (a) course deviation reaction times, (b) $\log$ (late arrival reaction times)

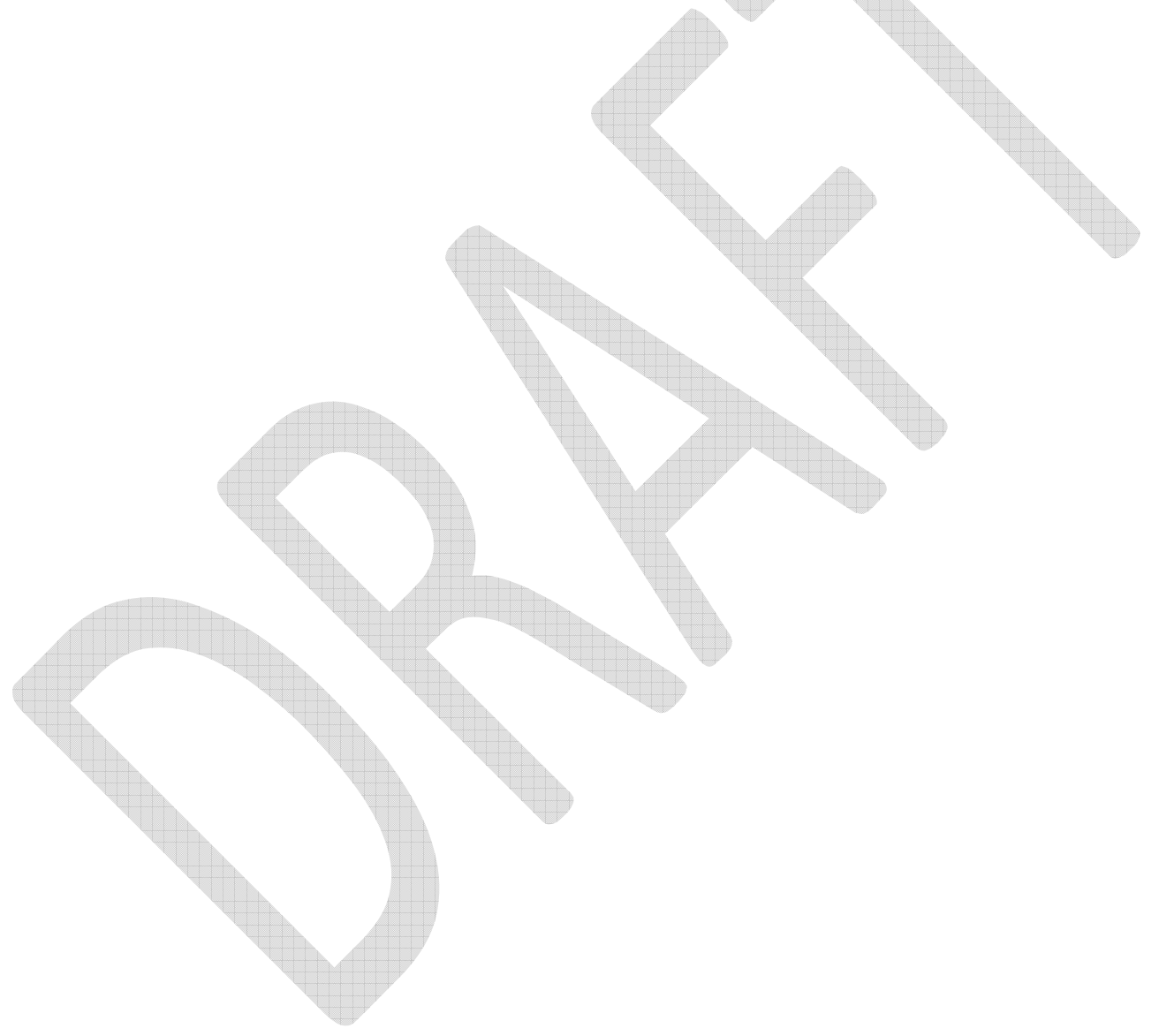




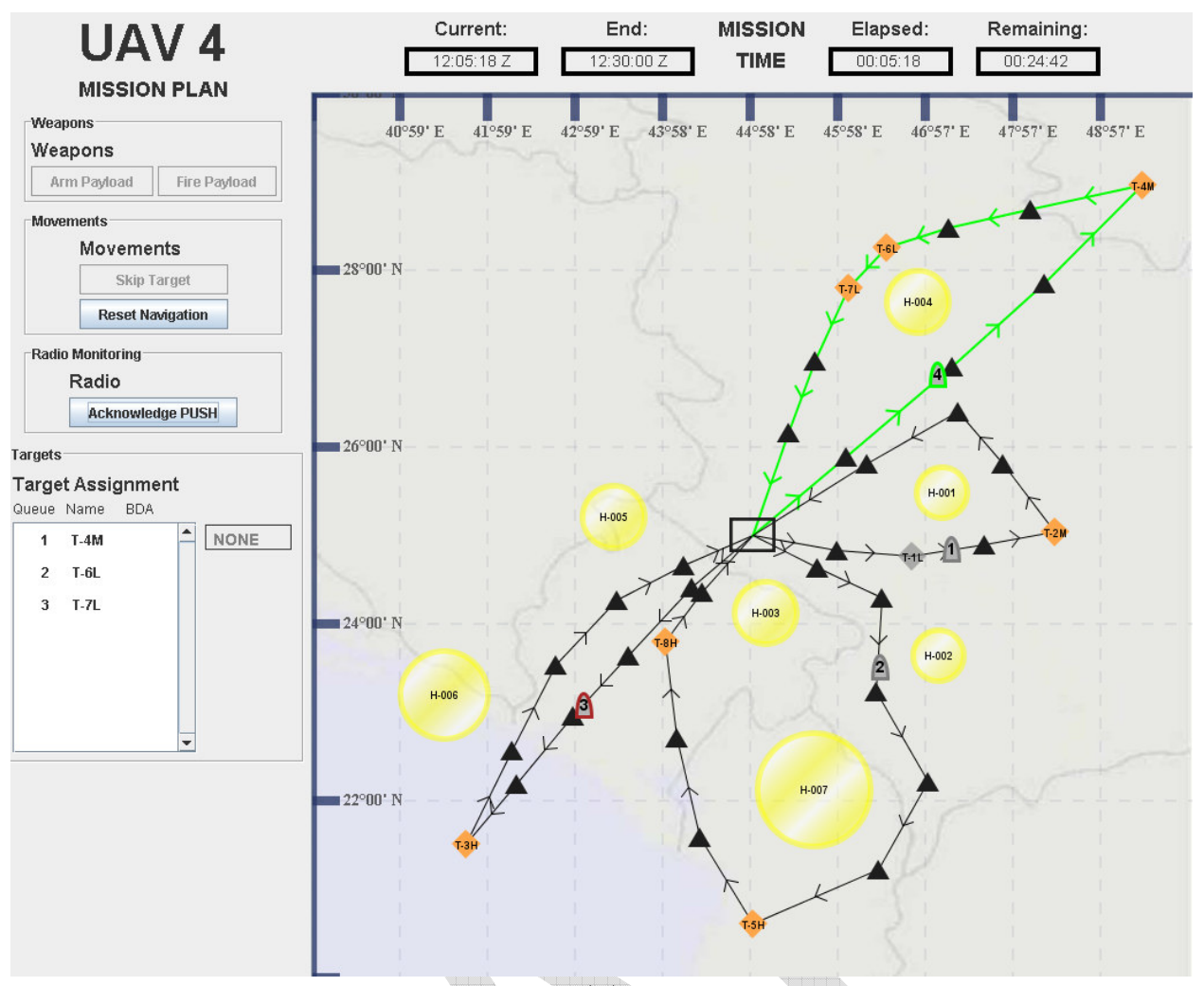

(a)

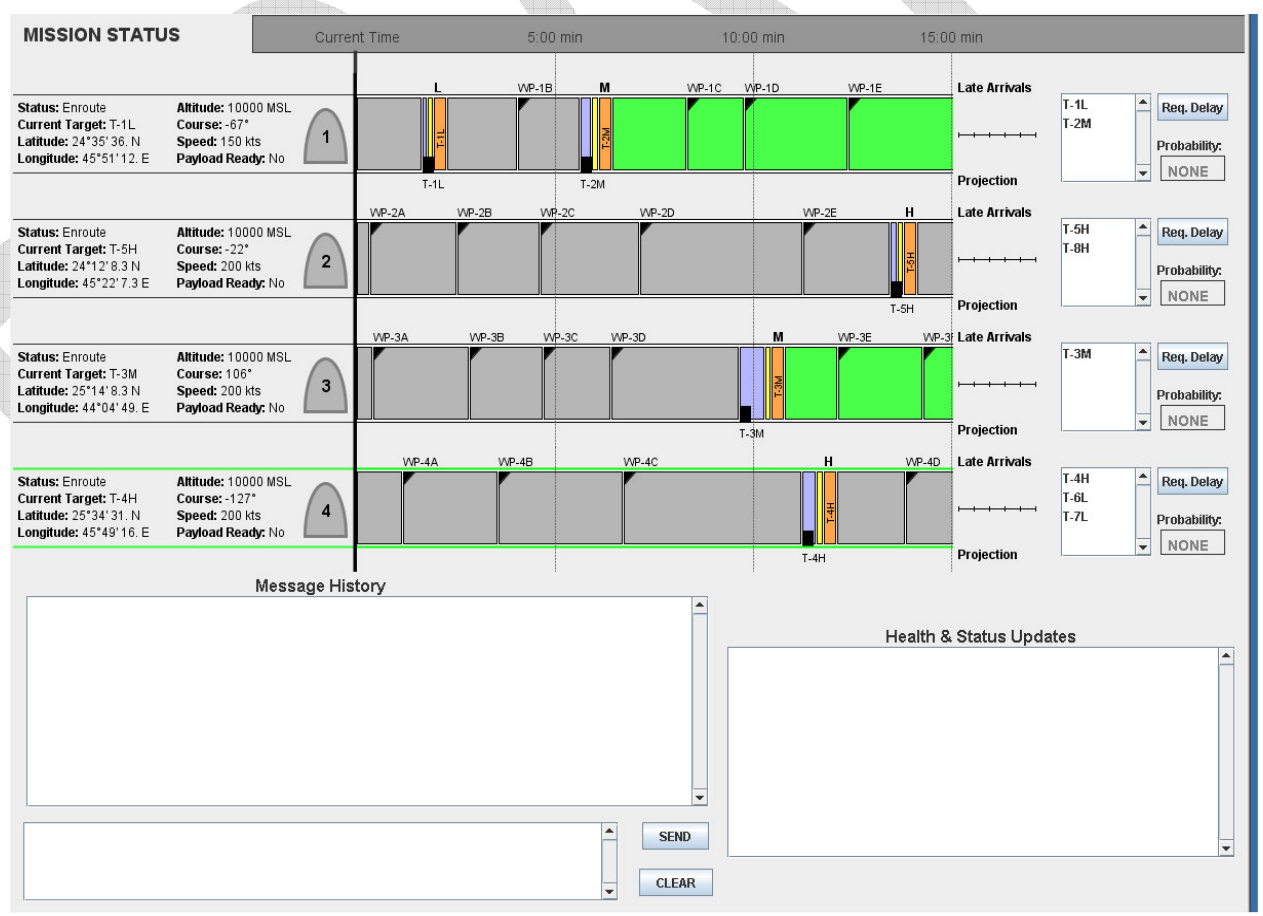

(b)

Figure 1. Multiple Autonomous Unmanned Vehicle Experimental (MAUVE) test bed (a) map display (b) timeline display 


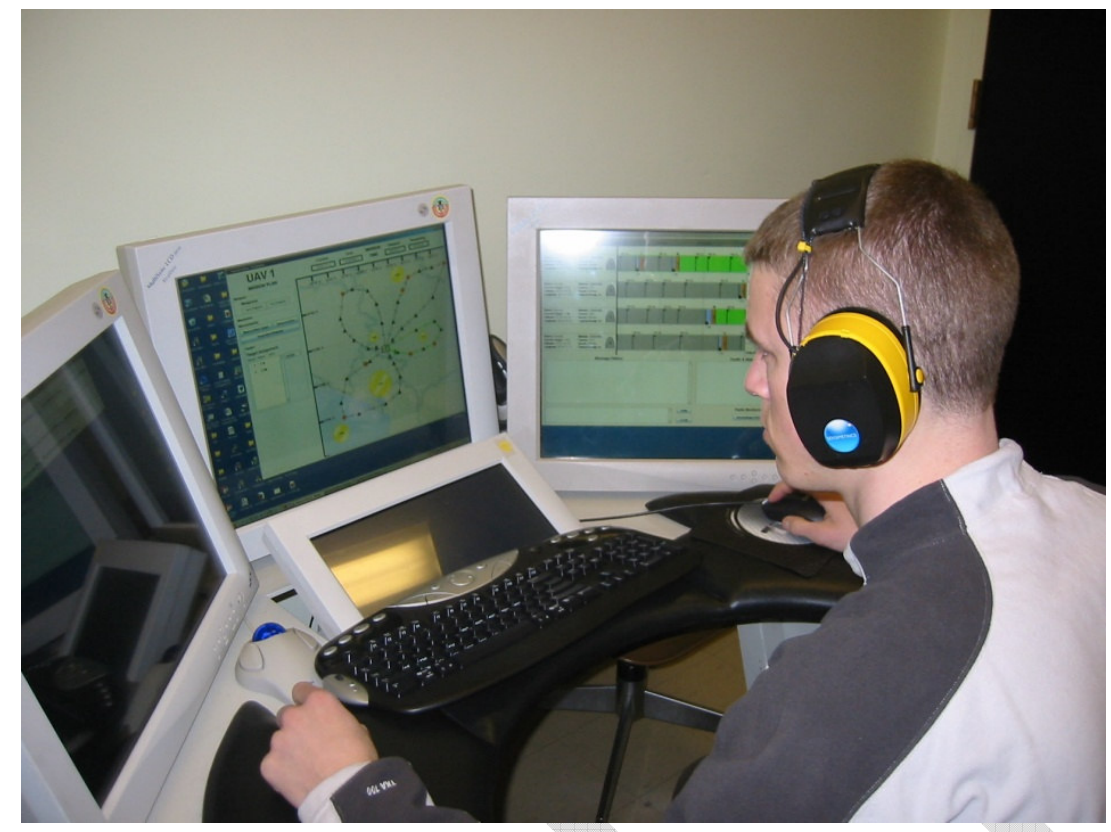

Figure 2. The multi-modal workstation 


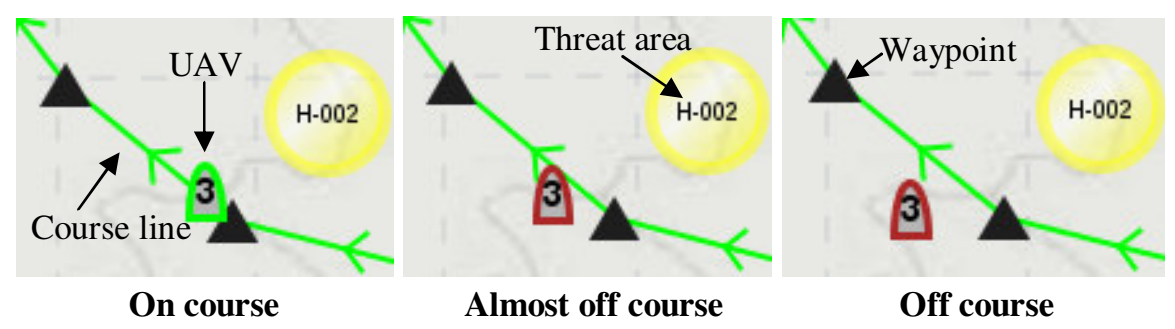

(a)

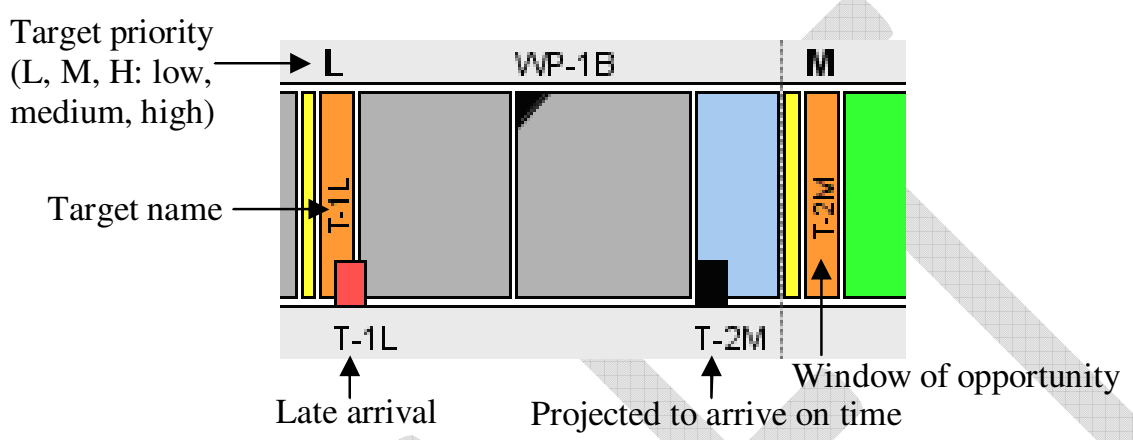

(b)

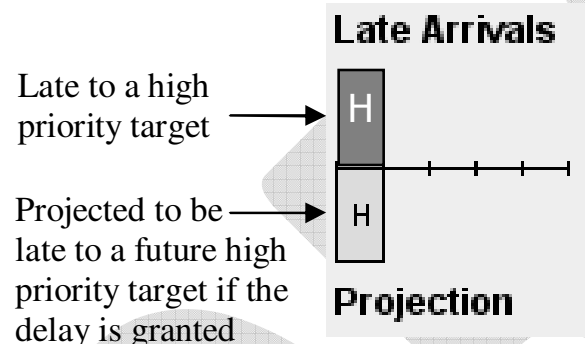
delay is granted

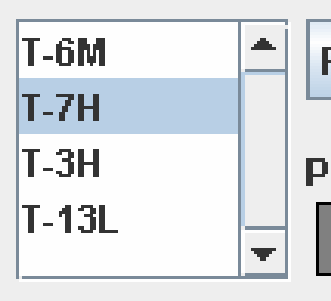

(c)

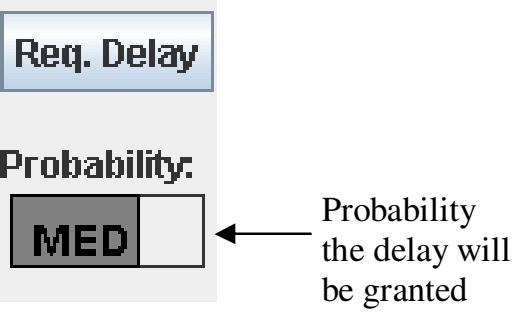

be granted

Figure 3. (a) A course deviation event (b) a late arrival event (c) decision support visualization for late arrivals 


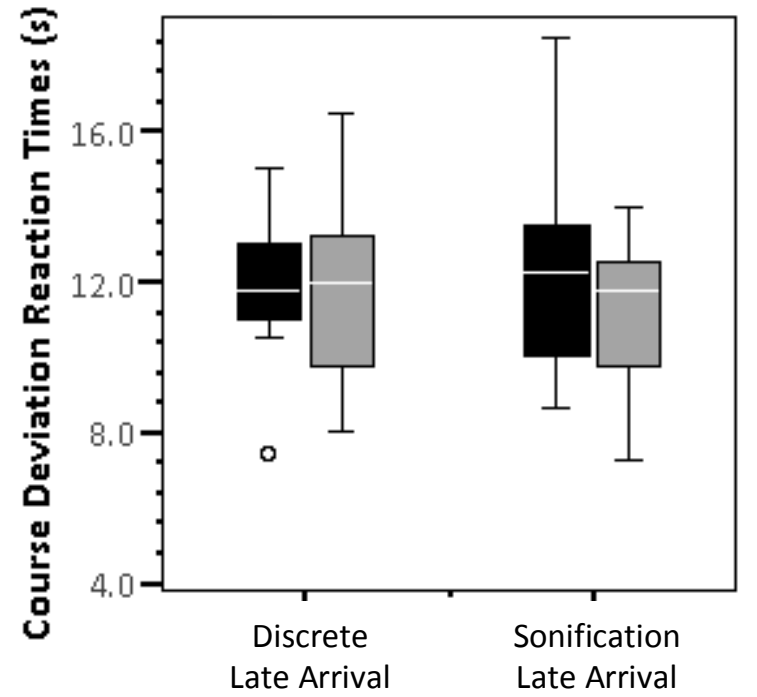

Discrete Course Deviation

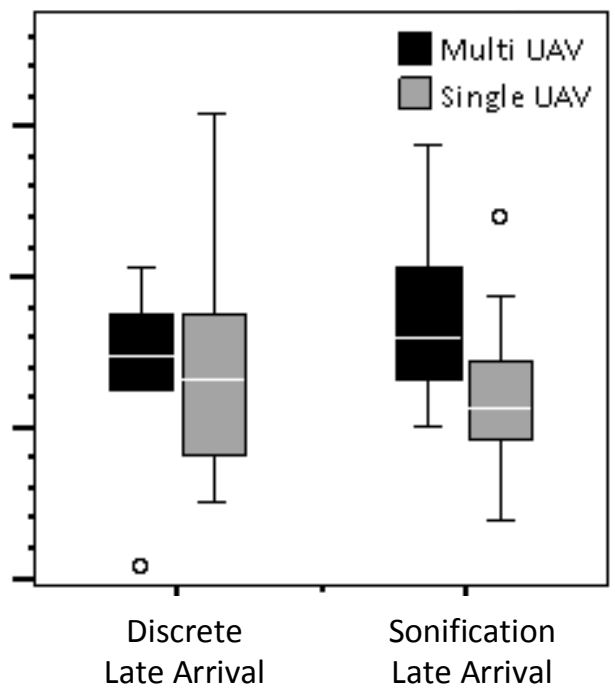

Sonification Course Deviation

\section{Auditory Alert Type}

(a)

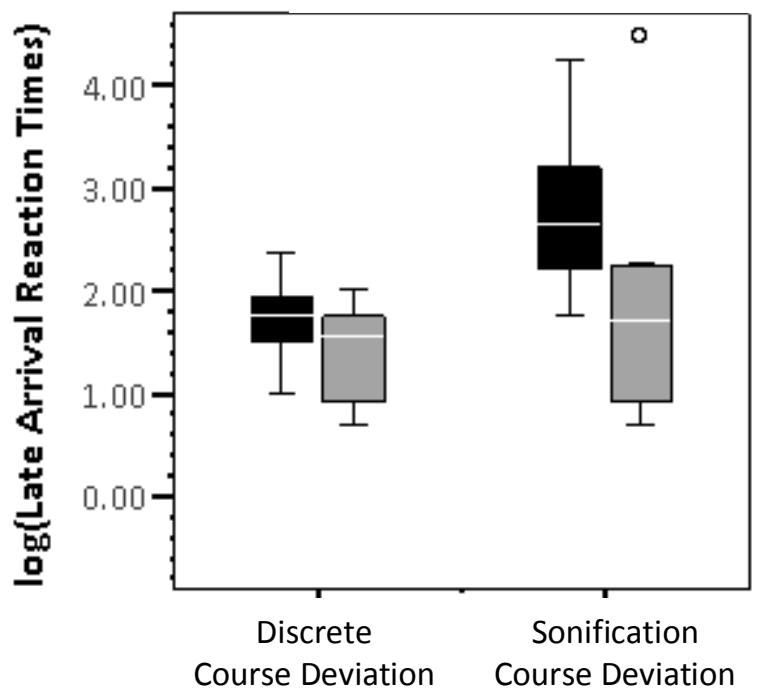

Discrete Late Arrival

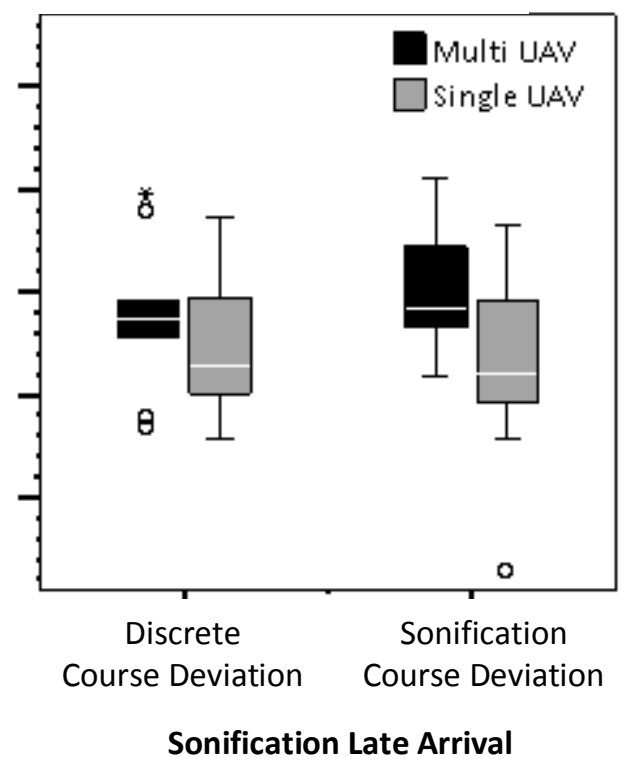

Sonification Late Arrival

\section{Auditory Alert Type}

(b)

Figure 4. Box plots for (a) course deviation reaction times, (b) $\log$ (late arrival reaction times) 


\section{BIOGRAPHIES}

Birsen Donmez is a postdoctoral associate with the Humans and Automation Laboratory of MIT. She has a Ph.D. (2007) and a M.S. (2004) in Industrial Engineering, as well as a M.S. (2007) in Statistics from the University of Iowa.

M. L. Cummings received her Ph.D. in Systems Engineering from the University of Virginia in 2003. She is currently an associate professor in the MIT Aeronautics \& Astronautics Department, and is the director of the Humans and Automation Laboratory.

Hudson D. Graham was a graduate research assistant at the Humans and Automation Laboratory of MIT from 2006 to 2008. He has a M.S. (2008) in Engineering Systems from MIT and a B.S. (2006) in Systems Engineering from the US Air Force Academy.

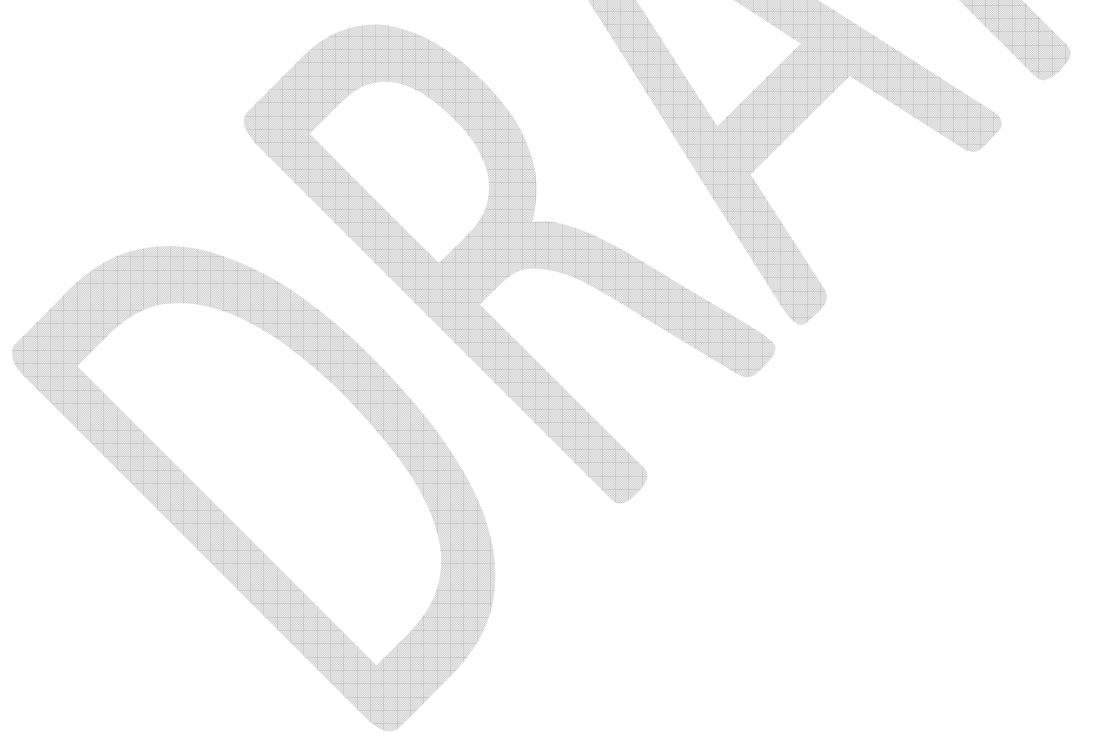

